\title{
Cross-neutralization of SARS-CoV-2 Kappa and Delta variants by inactivated vaccine-elicited serum and monoclonal antibodies
}

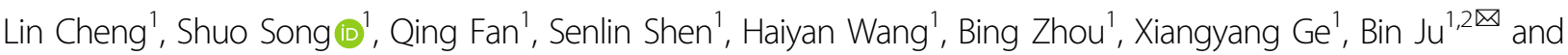 \\ Zheng Zhang $\mathbb{1}^{1,2,3 凶}$
}

Dear Editor,

The SARS-CoV-2 variant of B.1.617 lineage was first identified in India in October 2020, and rapidly spread to many other countries around the world. The variant includes three main sub-lineages, B.1.617.1 (Kappa), B.1.617.2 (Delta), and B.1.617.3 ${ }^{1}$. In light of their high transmissibility, the Kappa and Delta variants had been designated as a variant of interest and a variant of concern by the World Health Organization, respectively. As shown in Fig. 1a, the two variants shared L452R mutation in the receptor-binding domain (RBD). In addition, the variants also harbor unique mutations: E484Q in the Kappa variant and T478K in the Delta variant ${ }^{2}$.

SARS-CoV-2 utilized the RBD of viral spike protein to recognize its cellular receptor (angiotensin-converting enzyme 2, ACE2), and disruption of the RBD-ACE2 interaction could block virus entry, which was usually regarded as the candidate target of antiviral drugs and neutralizing antibodies (nAbs) ${ }^{3}$. Therefore, mutations that appeared in RBD may affect the viral infection and transmission, and may even lead to the escape of SARS$\mathrm{CoV}-2$ from the neutralization of nAbs elicited by natural virus infection and induced by vaccine ${ }^{4}$. The three mutation residues (L452, T478, and E484) were involved in or near the footprint of ACE2 on the RBD (Fig. 1b),

\footnotetext{
Correspondence: Bin Ju (jubin2013@163.com) or

Zheng Zhang (zhangzheng1975@aliyun.com)

${ }^{1}$ Institute for Hepatology, National Clinical Research Center for Infectious

Disease, Shenzhen Third People's Hospital; The Second Affiliated Hospital,

School of Medicine, Southern University of Science and Technology, Shenzhen,

Guangdong, China

${ }^{2}$ Guangdong Key Laboratory for Anti-Infection Drug Quality Evaluation,

Shenzhen, Guangdong, China

Full list of author information is available at the end of the article

These authors contributed equally: Lin Cheng, Shuo Song, Qing Fan
}

which may influence the binding affinity of ACE2 and neutralizing activity of nAbs.

It is widely known that RBD-specific nAbs could be divided into four classes according to the competition with ACE2 and the accessibility of recognizing epitopes on the RBD in 'up' or 'down' conformation (Fig. 1c) ${ }^{3}$. The three mutations (L452R, E484Q, and $\mathrm{T} 478 \mathrm{~K}$ ) that appeared in Kappa and Delta variants were located in and near the binding interface between RBD and most of the nAbs from Classes 1,2, and 3, indicating that these two variants may escape from the neutralization of nAbs. It was encouraging that nAbs of Class 4 recognized a distinct epitope away from the mutation region of SARSCoV-2 Kappa and Delta variants, which may still retain neutralizing activities (Fig. 1d).

Nowadays, various SARS-CoV-2 vaccines, including inactivated, mRNA, DNA, adenovirus vector, and recombination protein vaccines, have been developed to combat the viral infection ${ }^{5}$. The immunogenicity of vaccines usually varied in different platforms with distinct amounts of antigen and stimulation times. Even the same type of vaccines from different companies induced varying degrees of immune responses ${ }^{6}$. Although a recent smallsample study reported that the geometric mean titer (GMT) of neutralizing antibodies induced by the inactivated vaccine (Coronavac) was about tenfold lower than that induced by the mRNA vaccine (BNT162b2) ${ }^{7}$, inactivated vaccines still showed good immunogenicity and protective effect against SARS-CoV-2 in animal models and clinical trials ${ }^{8,9}$. Meanwhile, several studies have encouragingly revealed that despite a certain degree of decline, vaccinated sera preserved neutralizing activities against Alpha and Beta variants ${ }^{10}$. However, little is known about the protective efficacy of the inactivated 


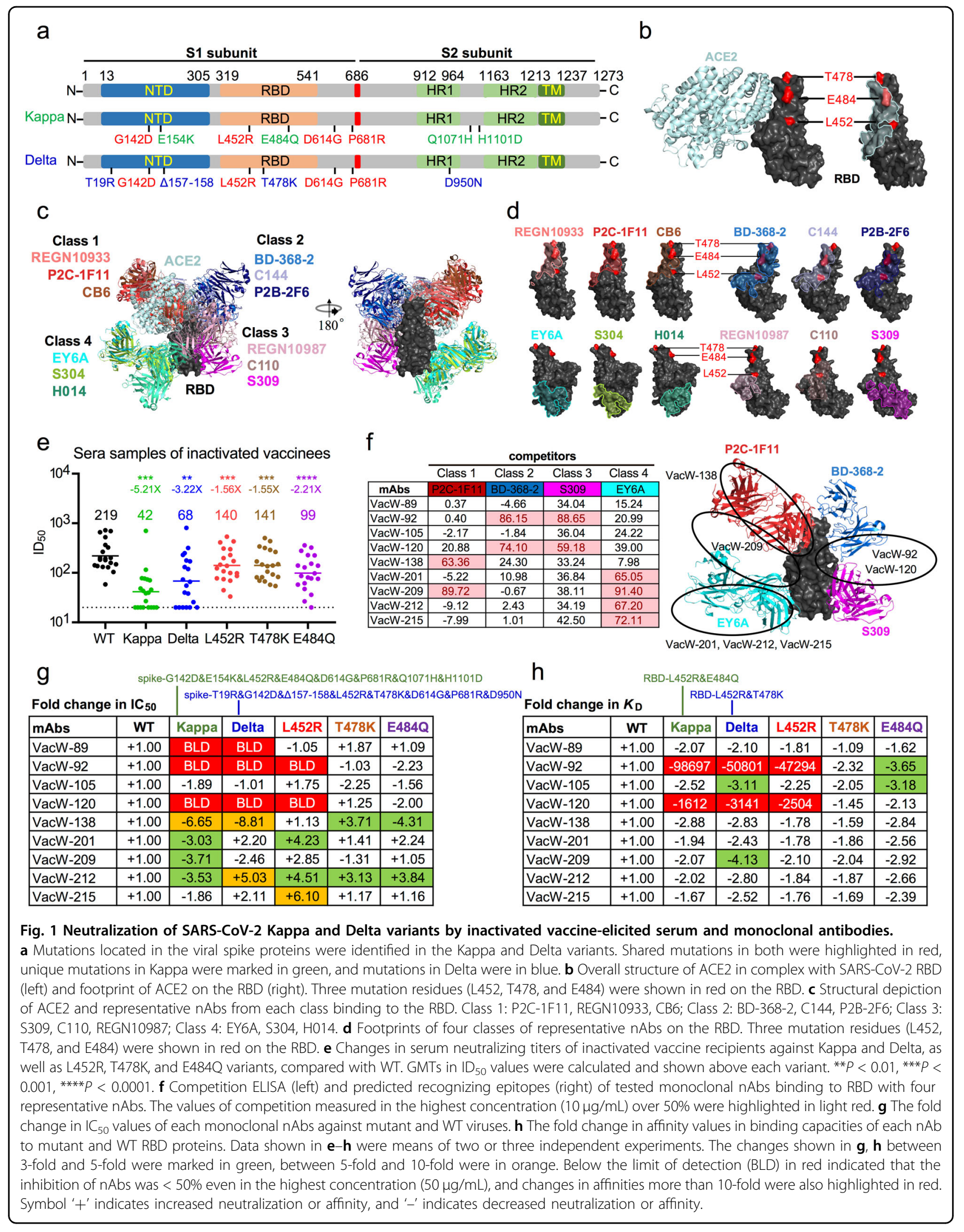


vaccine against Kappa and Delta variants. Especially, the cross-neutralization of monoclonal nAbs isolated from inactivated vaccine recipients has yet to be reported.

Here, we prepared a series of SARS-CoV-2 pseudoviruses bearing spike proteins of the Wuhan reference strain (wild type, WT), Kappa, or Delta variants, as well as L452R, E484Q, or T478K single mutations based on the HIV-1 backbone, which is widely accepted to evaluate the neutralizing activities of various serum samples and monoclonal $n A b s^{11,12}$. Twenty vaccinated serum samples with high values of RBD-specific binding antibodies collected at about 2 weeks after immunization with two doses of inactivated vaccine were incorporated into this study (Supplementary Table S1). We first measured the neutralizing activities of these serum samples against WT, Kappa, and Delta variants (Supplementary Fig. S1). The levels of neutralizing antibodies against Kappa and Delta variants were lower than those against WT, which significantly displayed a 5.21 -fold reduction (42 vs 219 ) and a 3.22-fold decline (68 vs 219 ) respectively in GMTs (Fig. 1e and Supplementary Fig. S2a). We further evaluated the influence of three single mutations present in RBD of variants on their escape from polyclonal nAbs, and the neutralizing activities of serum samples against L452R, $\mathrm{T} 478 \mathrm{~K}$, and $\mathrm{E} 484 \mathrm{Q}$ variants were reduced by $1.56-, 1.55-$, and 2.21-fold compared to those against WT (Fig. 1e and Supplementary Fig. S2a). These data indicated that SARSCoV-2 Kappa and Delta variants reduced their sensitivities to the inactivated vaccine-elicited serum to some extent. In addition, the neutralizing potency of serum against variants was strongly related to that against WT strain (Supplementary Fig. S2b).

Two groups have reported the neutralizing activities of monoclonal nAbs isolated from volunteers who received mRNA or inactivated vaccines, suggesting that vaccines could effectively induce nAbs against WT SARS-CoV-2 and several variants ${ }^{12,13}$. However, it is poorly understood whether Kappa and Delta variants escape from the neutralization of monoclonal nAbs elicited by inactivated vaccine. Here, we used the SARS-CoV-2 RBD protein as a bait to sort specific single B cells by flow cytometry from two volunteers (Supplementary Fig. S3). Nine monoclonal nAbs were isolated and characterized, whose neutralizing potencies ranged from 0.03 to $11.11 \mu \mathrm{g} / \mathrm{mL}$ against WT (Supplementary Figs. S4 and S6a). The gene usages of these nAbs were derived from multiple germlines including IGHV1-46, 1-69, 3-23, 3-7, etc. (Supplementary Table S2). Importantly, the heavy chains of VacW-209 and VacW-215 belonged to IGHV3-30 and 3-53 germlines, respectively, which were also termed as public antibodies and usually shared among COVID-19 patients $^{14,15}$. More encouragingly, VacW-209 was a potent neutralizer with $\mathrm{IC}_{50}$ of $0.03 \mu \mathrm{g} / \mathrm{mL}$ similar to that of P2C-1F11 identified from a COVID-19 patient, the
Fc-modified version (named Brii-196) of which is being investigated in phase 3 of the clinical trial. In addition, the neutralizing activity of VacW-209 was more potent than previously reported nAbs isolated from inactivated vaccinees ${ }^{13}$.

Using competition ELISA, we predicted the binding epitopes of these nine nAbs based on their competition with four representative nAbs. As shown in Fig. If and Supplementary Fig. S5, VacW-92 and VacW-120 were classified into Class $2 / 3$, VacW-138 recognized the epitope of Class 1, VacW-201, VacW-212, and VacW-215 belonged to Class 4 nAbs, and VacW-209 exhibited high competition with both P2C-1F11 (Class 1) and EY6A (Class 4) of binding to RBD. We failed to predict the recognizing epitopes of VacW-89 and VacW-105 due to their low binding affinities. It was also possible that these two nAbs might recognize some novel epitopes beyond the four classes described above. Overall, these results demonstrated that the inactivated vaccine induced a similar RBD-specific antibody response to natural SARSCoV-2 infection.

Finally, we further tested the cross-neutralizing and binding activities of these nine nAbs against SARS-CoV-2 Kappa and Delta variants, as well as several single mutations (Supplementary Figs. S4 and S6a). Most of the nAbs still maintained their neutralizing activities despite some with slight reductions, except for VacW-89, VacW-92, and $\mathrm{VacW}-120$, whose inhibitions were below $50 \%$ when tested at the highest concentration $(50 \mu \mathrm{g} / \mathrm{mL})$ (Fig. $1 \mathrm{~g}$ ). Of note, VacW-209 effectively neutralized all tested variants, including Kappa, Delta, L452R, T478K, and E484Q, with similar potencies to that of the above-mentioned P2C-1F11 (Supplementary Fig. S4). The neutralizing activities of monoclonal nAbs decreased with the same trend as those of the two vaccinee serum samples, with obvious reductions for Kappa and Delta variants and moderate alterations for single point mutations. Consistently, the binding affinities of these nAbs to WT and mutant RBD proteins (RBD-WT, L452R\&E484Q, L452R\&T478K, L452R, T478K, and E484Q) displayed the same pattern with their neutralizing activities (Fig. $1 \mathrm{~h}$ and Supplementary Figs. S6b, S7 and Table S3), indicating a strong correlation between binding avidity and neutralization. VacW-92 and VacW-120 bound very weakly to the mutant RBD proteins bearing L452R, which revealed the escape mechanism of SARS-CoV-2 variants from the neutralization of antibodies.

In conclusion, we comprehensively reported the neutralization of serum antibodies elicited by the inactivated vaccine against SARS-CoV-2 Kappa and Delta variants. The cross-neutralization of serum samples against mutant viruses partially decreased compared with that against WT, and the mutation E484Q resulted in a more significant decline of neutralizing activity than L452R and 
T478K. These findings were consistent with other studies showing similar loss of neutralization of serum samples from convalescent patients or induced by mRNA or adenovirus vector vaccines ${ }^{1,2}$. Although it does exist a reduction in the neutralization of inactivated vaccineelicited sera, they really maintained some degree of protective immunity against the infection of mutant viruses, especially the Delta variant, which is still raging across the world and causes more serious disease processes. Encouragingly, we isolated and characterized a series of monoclonal nAbs from inactivated vaccine recipients, whose germline gene usages, recognition of epitopes, neutralizing potencies, and cross-neutralization against SARS-CoV-2 variants were similar to those nAbs elicited by natural virus infection. Taken together, our results emphasized the importance of the immunization of inactivated vaccine to prevent infection of Kappa and Delta variants, which could contribute to controlling the pandemic. We have also proved the ability of the inactivated vaccine to induce broadly monoclonal nAbs and identified a potent neutralizer VacW-209 which could serve as a candidate therapeutic antibody in eliciting passive protection against various SARS-CoV-2 variants.

\section{Acknowledgements}

We thank all the participants who received the inactivated vaccine and all the healthy providers from Shenzhen Third People's Hospital for their inputs. This study was supported by the National Science Fund for Distinguished Young Scholars (82025022), the National Natural Science Foundation of China (82002140), the Guangdong Basic and Applied Basic Research Foundation (2021B1515020034, 2019A1515011197, 2021A1515011009, 2020A1515110656), and the Shenzhen Science and Technology Program (RCYX20200714114700046, JSGG20200207155251653, JSGG20200807171401008, KQTD20200909113758004, JCYJ20190809115617365, JCYJ20180228162229889).

\section{Author details}

'Institute for Hepatology, National Clinical Research Center for Infectious Disease, Shenzhen Third People's Hospital; The Second Affiliated Hospital, School of Medicine, Southern University of Science and Technology, Shenzhen, Guangdong, China. ${ }^{2}$ Guangdong Key Laboratory for Anti-Infection Drug Quality Evaluation, Shenzhen, Guangdong, China. ${ }^{3}$ Shenzhen Research Center for Communicable Disease Diagnosis and Treatment of Chinese Academy of Medical Science, Shenzhen, Guangdong, China

\section{Author contributions}

Z.Z. is the principal investigator of this study. Z.Z. and B.J. conceived and designed the study. L.C., S. Song, Q.F., and B.J. performed all experiments together with assistance from H.W., B.Z., and X.G., S. Shen summarized the structural information of published nAbs and analyzed their binding modes and footprints on the RBD. Z.Z. and B.J. wrote the manuscript and all authors read and approved the manuscript.

\section{Conflict of interest}

The authors declare no competing interests.

\section{Publisher's note}

Springer Nature remains neutral with regard to jurisdictional claims in published maps and institutional affiliations.

Supplementary information The online version contains supplementary material available at https://doi.org/10.1038/s41421-021-00347-1.

Received: 22 August 2021 Accepted: 30 September 2021

Published online: 23 November 2021

\section{References}

1. Planas, D. et al. Reduced sensitivity of SARS-CoV-2 variant Delta to antibody neutralization. Nature 596, 276-280 (2021).

2. Liu, C. et al. Reduced neutralization of SARS-CoV-2 B.1.617 by vaccine and convalescent serum. Cell 184, 4220-4236 (2021).

3. Barnes, C. O. et al. SARS-CoV-2 neutralizing antibody structures inform therapeutic strategies. Nature 588, 682-687 (2020).

4. Chen, R. E. et al. Resistance of SARS-CoV-2 variants to neutralization by monoclonal and serum-derived polyclonal antibodies. Nat. Med. 27, 717-726 (2021).

5. Subbarao, $K$. The success of SARS-CoV-2 vaccines and challenges ahead. Cell Host Microbe 29, 1111-1123 (2021).

6. Dai, L. et al. Viral targets for vaccines against COVID-19. Nat. Rev. Immunol. 21, 73-82 (2021).

7. Lim, W. W. et al. Comparative immunogenicity of mRNA and inactivated vaccines against COVID-19. Lancet Microbe 2, e423 (2021).

8. Wang, H. et al. Development of an inactivated vaccine candidate, BBIBP-CorV, with potent protection against SARS-CoV-2. Cell 182, 713-721 (2020).

9. Xia, S. et al. Safety and immunogenicity of an inactivated SARS-CoV-2 vaccine, BBIBP-CorV: a randomised, double-blind, placebo-controlled, phase 1/2 trial. Lancet Infect. Dis. 21, 39-51 (2021).

10. Wang, G. L. et al. Susceptibility of circulating SARS-CoV-2 variants to neutralization. N. Engl. J. Med. 384, 2354-2356 (2021).

11. Wang, R. et al. Analysis of SARS-CoV-2 variant mutations reveals neutralization escape mechanisms and the ability to use ACE2 receptors from additional species. Immunity 54, 1611-1621 (2021).

12. Wang, Z. et al. mRNA vaccine-elicited antibodies to SARS-CoV-2 and circulating variants. Nature 592, 616-622 (2021).

13. Zhou, X. et al. Molecular deconvolution of the neutralizing antibodies induced by an inactivated SARS-CoV-2 virus vaccine. Protein Cell 12, 818-823 (2021).

14. Robbiani, D. F. et al. Convergent antibody responses to SARS-CoV-2 in convalescent individuals. Nature 584, 437-442 (2020).

15. Zhang, Q. et al. Potent and protective IGHV3-53/3-66 public antibodies and their shared escape mutant on the spike of SARS-CoV-2. Nat. Commun. 12 4210 (2021). 